\title{
A Novel Continuous and Structural VAR Modeling Approach and Its Application to Reactor Noise Analysis
}

\author{
MARINA DEMESHKO, TAKASHI WASHIO, and YOSHINOBU KAWAHARA, \\ Osaka University \\ YURIY PEPYOLYSHEV, Joint Institute of Nuclear Research
}

\begin{abstract}
A vector autoregressive model in discrete time domain (DVAR) is often used to analyze continuous time, multivariate, linear Markov systems through their observed time series data sampled at discrete timesteps. Based on previous studies, the DVAR model is supposed to be a noncanonical representation of the system, that is, it does not correspond to a unique system bijectively. However, in this article, we characterize the relations of the DVAR model with its corresponding Structural Vector AR (SVAR) and Continuous Time Vector AR (CTVAR) models through a finite difference method across continuous and discrete time domain. We further clarify that the DVAR model of a continuous time, multivariate, linear Markov system is canonical under a highly generic condition. Our analysis shows that we can uniquely reproduce its SVAR and CTVAR models from the DVAR model. Based on these results, we propose a novel Continuous and Structural Vector Autoregressive (CSVAR) modeling approach to derive the SVAR and the CTVAR models from their DVAR model empirically derived from the observed time series of continuous time linear Markov systems. We demonstrate its superior performance through some numerical experiments on both artificial and real-world data.
\end{abstract}

Categories and Subject Descriptors: C.1.16 [Data Mining and Knowledge Discovery]: Systems and Applications

General Terms: Casual Discovery, ARMA Models, Control Theory

Additional Key Words and Phrases: VAR model, SVAR model, CTVAR model, continuous time linear Markov system, canonicality, nuclear reactor noise analysis

ACM Reference Format:

Marina Demeshko, Takashi Washio, Yoshinobu Kawahara, and Yuriy Pepyolyshev. 2015. A novel continuous and structural VAR modeling approach and its application to reactor noise analysis. ACM Trans. Intell. Syst. Technol. 7, 2, Article 24 (November 2015), 22 pages.

DOI: http://dx.doi.org/10.1145/2710025

\section{INTRODUCTION}

Many real-world systems are well represented by continuous time, multivariate, linear Markov systems. Usually they are observed as time series data sampled at discrete timesteps for digital processing. In the case when such objective system is stable ${ }^{1}$ and

\footnotetext{
${ }^{1}$ The system is stable if all nearby initial conditions converge to the equilibrium point.
}

Authors' addresses: M. Demeshko, T. Washio, and Y. Kawahara, The Institute of Scientific and Industrial Research, Osaka University, 8-1, Mihogaoka, Ibaraki City, Osaka, 567-0047, Japan; emails: marina.p.dem@ gmail.com, \{washio, kawahara\}@ar.sanken.osaka-u.ac.jp; Y. Pepyolyshev, Frank Laboratory of Neutron Physics, JINR, Joliot-Curie 6, Dubna, Moscow Region, 141980, Russia; email: pepel@nf.jinr.ru.

Permission to make digital or hard copies of all or part of this work for personal or classroom use is granted without fee provided that copies are not made or distributed for profit or commercial advantage and that copies bear this notice and the full citation on the first page. Copyrights for components of this work owned by others than the author(s) must be honored. Abstracting with credit is permitted. To copy otherwise, or republish, to post on servers or to redistribute to lists, requires prior specific permission and/or a fee. Request permissions from permissions@acm.org.

2015 Copyright is held by the owner/author(s). Publication rights licensed to ACM.

2157-6904/2015/11-ART24 $\$ 15.00$

DOI: http://dx.doi.org/10.1145/2710025 
controllable, ${ }^{2}$ a Discrete time Vector Autoregressive (DVAR) ${ }^{3}$ model is often used to analyze it [Brockwell and Davis 1991]. However, according to previous studies [Stamer et al. 1996; Gottschalk 2001], the DVAR model is not canonical, that is, it does not have a bijective correspondence with the objective system for which it is derived. This creates difficulties in identifying the structure and the parameters of the objective system dynamics.

As a remedy to this limitation of the DVAR modeling, a Structural Vector AR (SVAR) model [Moneta et al. 2010] has been studied. The SVAR model has a bijective correspondence with a unique system. It is used for modeling when an objective continuous time linear Markov system is exactly described by an AR process. Moreover, the SVAR model provides information on the propagation of influences among variables in the system, even if the DVAR is only an approximation of the objective dynamic system. However, in the past studies, some strong assumptions were required on the system structure and/or variables for the identification of the SVAR model. These assumptions are acyclic dependency among variables and non-Gaussianity of external noises [Hyvarinen et al. 2008], orthogonality and nonlinear restrictions on parameter matrices of the external noises [Gottschalk 2001; Pfaff and Kronberg 2008; Kilian 2011], and recursive ordering of system parameters [Gottschalk 2001; Kilian 2011]. They largely limit the applicability of the SVAR modeling in the system structure analysis.

Based on these considerations, this study aims to achieve the following four objectives.

(1) Show under a generic assumption, that the DVAR model of a continuous time, multivariate, linear Markov system is canonical.

(2) Clarify mathematical relations among a Continuous Time Vector AR (CTVAR) model, a SVAR model and a DVAR model of the system.

(3) Present a new approach named Continuous time Structural Vector Autoregressive (CSVAR) modeling for the CTVAR and the SVAR models based on the DVAR model obtained from observed time series data.

(4) Demonstrate using numerical experiments on artificial and real-world time series the applicability and the accuracy of the proposed approach.

This article is organized as follows. Section 2 presents past studies related with our work and clarifies technical issues to be addressed. In Section 3, we established a new principle to reconstruct the CTVAR and SVAR models from the DVAR model estimated from observed time series. In Section 4, we show a performance of our proposed approach by numerical experiments using artificial and real-world data and its comparison with a past representative SVAR modeling approach.

\section{PRELIMINARY DISCUSSION AND ANALYSIS}

\subsection{Related Work}

A DVAR model, Equation (1), of order $p$ is a general representation of a stable, controllable, multivariate, liner Markov system with a given $d$-dimensional variable vector $Y(t)$ observed with a discrete timestep $\Delta t$.

$$
Y(t)=\sum_{j=1}^{p} \Phi_{j} Y(t-j \Delta t)+U(t)
$$

\footnotetext{
${ }^{2}$ The system is controllable if its current state can be transferred to any given state by applying an appropriate input series over a finite time period.

${ }^{3}$ In the previous studies, the notion of a vector autoregressive model (VAR) is usually used as a discrete time model. In our article, to separate the vector autoregressive processes for discrete and continuous time domains, we will use the term of a discrete time vector autoregressive (DVAR) model instead.
} 
where $\Phi_{j}$ are $d \times d$ coefficient matrices. $U(t)$ is a $d$-dimensional unobserved noise vector that is independent and identically distributed (i.i.d.) in a discrete time domain [Brockwell and Davis 1991]. This model has some ambiguity to represent infinitely many systems, since Equation (1) is equivalent to

$$
Y(t)=\sum_{j=1}^{p} \Phi_{j} Y(t-j \Delta t)+Q W(t),
$$

where $Q$ is a $d \times d$ invertible matrix and $W(t)=Q^{-1} U(t)$. Thus, depending on the choices of $Q, Y(t)$ has infinitely many impulse responses for $W(t)$ [Moneta et al. 2010]. In other words, for a given observed time series, $Y(t)$, the infinite number of systems that could generate $Y(t)$ are represented by a unique DVAR model with parameters $\Phi_{j}$, $j=1 \ldots p$. Therefore, the DVAR model has no bijective correspondence to the objective system, that is, it is not canonical. Thus, we need to know a unique combination of $Q$ and $W(t)$ that corresponds to the objective system dynamics in a bijective manner to identify the system uniquely. Having such $Q$ and $W(t)$, Equation (2) is further rewritten as

$$
Y(t)=\sum_{j=0}^{p} \Psi_{j} Y(t-j \Delta t)+W(t),
$$

where $\Psi_{0}=I-Q^{-1}, \Psi_{j}=Q^{-1} \Phi_{j}$. This equation includes fast effects in its representation by a matrix $\Psi_{0}$ representing a feedback of $Y(t)$ on itself. If there are no such effects in the objective system, then $\Psi_{0}$ should be a zero matrix, otherwise $\Psi_{0}$ is nonzero and $Q \neq I$. By deriving a unique matrix $\Psi_{0}$, we define the unique combination of $Q$ and $W(t)$ in Equation (3), which provides a unique model with parameters $\Psi_{j}, j=0, \ldots, p$ that bijectively represents the unique objective system. Therefore, this model is canonical. In the past studies, Equation (3) is called a SVAR model [Moneta et al. 2010; Kawahara et al. 2011] .

The SVAR model is provided by the derivation of the unique $\Psi_{0}$. As shown in Equation (3), matrices $\Psi_{0}$ and $\Psi_{j}$ directly depend on $Q$. However, matrix $Q$ is not reproduced from the DVAR model, because it is integrated in $\Phi_{j}$ as $\Phi_{j}=Q \Psi_{j}$. Accordingly, we need some extra information to provide $Q$. However, even if we introduce an orthonormality constraint $Q Q^{T}=E_{t}\left(U(t) U(t)^{T}\right)$ to make noises in $W(t)$ mutually uncorrelated, the representation of the DVAR model given by this approach is not unique, because there are many choices for $Q$ that satisfy that constraint, for example, $Q O$ satisfying $Q O(Q O)^{T}=Q O O^{T} Q^{T}=Q Q^{T}$, where $O$ is any orthonormal matrix [Moneta et al. 2011].

There are many studies on this issue for identifying the SVAR model. Most of these studies require strong assumptions on the objective system. For example, a study by Gottschalk [2001] introduced a constraint named exclusion restriction together with the orthonormality of the external noises. It requires some domain knowledge of the objective system to obtain the order of the noises in $U(t)$ and hence their corresponding variables in $Y(t)$. The order of the noises is further used for Cholesky decomposition that uniquely derives a strictly lower triangular $Q$. A study by Kilian [2011] introduced another constraint named a sign restriction. It also requires some domain knowledge on the signs of some elements of $Q$ with the aforementioned orthonormality of the noise vector to select the matrix $Q$. Such $Q$ is searched by testing the sign restriction after randomly generating orthonormal matrices with $Q R$ decompositions. A study by Hyvarinen et al. [2008] introduced less domain specific assumptions on the system. It proposed a novel method named AR-LiNGAM by assuming acyclic dependency among variables in $Y(t)$ and non-Gaussianity of external noises in $W(t)$ in addition to mutual 
independence and temporal uncorrelation of $W(t)$. The ordering information of the noises in $W(t)$ and their corresponding variables in $Y(t)$ were further provided by the application of Independent Component Analysis (ICA) to least-square residual noises of the DVAR modeling.

However, in many real-world applications, the aforementioned assumptions on the objective system significantly limit the applicability of these approaches, since such firm domain knowledge is not readily available. In this regard, we require a more generic approach to the SVAR modeling without using strong assumptions. To address this issue, we propose a novel modeling approach that uses only a very general assumption that subsumes stability and controllability of the objective system.

\subsection{Canonicality of VAR Models}

In Pearl [2000], Pearl stated that each noise in a canonical model has its own unique variable to directly and instantly change within a negligibly short time period required by attaining the equilibrium. Similar characterization and analysis of the canonical models can be seen in literature [Fisher 1970; Iwasaki and Simon 1994; Lacerda et al. 2008; Mooij et al. 2013]. $W(t)$ in the SVAR model, Equation (3), holds the characteristics of the noise vector in the canonical model, because each noise in $W(t)$ has its unique variable in $Y(t)$ to directly and instantly change. The term of $\Psi_{0}$ represents fast effects in $Y(t)$ that occur within the sampling time interval $\Delta t$. The other AR terms represent components generated by the system dynamics within a finite time interval, if the system is stable. Accordingly, the SVAR model is canonical under a given combination of invertible matrix $Q$ and $W(t)$ that correspond to the objective system.

Comparing the DVAR and the SVAR models, we obtain the following relations among their matrices and vectors [Kawahara et al. 2011; Hyvarinen et al. 2008].

$$
\begin{gathered}
\Phi_{j}=\left(I-\Psi_{0}\right)^{-1} \Psi_{j}, \\
\Psi_{j}=\left(I-\Psi_{0}\right) \Phi_{j}, \\
U(t)=\left(I-\Psi_{0}\right)^{-1} W(t), \\
W(t)=\left(I-\Psi_{0}\right) U(t) .
\end{gathered}
$$

We see that given a combination of $\Phi_{j}(j=1, \ldots, p)$, there are various combinations of $\Psi_{j}(j=1, \ldots, p)$, which induce the combination of $\Phi_{j}(j=1, \ldots, p)$ through Equation (4a). Thus, there exist multiple SVAR models, which induce the DVAR model. On the other hand, given a combination of $\Psi_{0}, \Psi_{j}(j=1, \ldots, p), Q$ is uniquely provided by $Q=\left(I-\Psi_{0}\right)^{-1}$, and a unique combination of $\Phi_{j}(j=1, \ldots, p)$ is also provided through Equation (4a). Thus, a given SVAR model induces only a unique combination of the DVAR model and the invertible matrix $Q$. In other words, the correspondence from the SVAR model to the DVAR model is surjective. In this sense, the DVAR model is not canonical.

On the other hand, when a stable, controllable, multivariate, linear Markov system in a continuous time domain is approximated by an AR process, it is represented by a Continuous Time Vector AR (CTVAR) model in Equation (6) consisting of continuous time stochastic differential equations with noise terms [Stamer et al. 1996].

$$
Y^{(p)}(t)=\sum_{m=0}^{p-1} S_{m} Y^{(m)}(t)+W(t),
$$

where $Y(t)$ and $W(t)$ are a $d$-dimensional observed variable vector and a $d$-dimensional external noise vector that is i.i.d. in a continuous time domain, respectively. $Y^{(m)}(t)$ is 
the $m$-th time differential of $Y(t)\left(=Y^{(0)}(t)\right)$, and $S_{m}(m=0, \ldots, p-1)$ is a $d \times d$ AR matrix [Stamer et al. 1996]. Since this is a continuous time model, it includes the fast effects in $Y(t)$ that occur within a period smaller than $\Delta t$. In this model, each noise in $W(t)$ directly and instantly changes the highest time differential of its corresponding unique variable in $Y(t)$, and the change propagates to the other lower order time differentials. In this regard, $W(t)$ satisfies the character of the noise vector in a canonical model. Additionally, $W(t)$ in Equation (6) is a unique noise vector, because application of $E(t)=P^{-1} W(t)$ with a $d \times d$ invertible matrix $P$ transforms Equation (6) as

$$
Y^{(p)}(t)=\left(I-P^{-1}\right) Y^{(p)}(t)+\sum_{m=0}^{p-1} P^{-1} S_{m} Y^{(m)}(t)+E(t) .
$$

Because any instantaneous process to change the highest order differentials $Y^{(p)}$ by itself is not admissible in a complete process dynamics, $P \neq I$ is not admitted. Thus, $W(t)$ is unique. This is because of the fact that the CTVAR model is an exact representation of the continuous AR processes in the objective system and thus includes all process dynamics. Accordingly, the CTVAR model, Eqation (6), is canonical, and the SVAR model, Equation (3), and the CTVAR model, Equation (6), bijectively correspond to the system dynamics. Since they represent the same stable, multivariate linear Markov system, which is controllable in both continuous and discrete time domains, the SVAR and the CVAR models have a bijective correspondence.

\section{PROPOSED PRINCIPLE}

In this section, we concentrate on objectives (1), (2), and (3) stated in Section 1. First, we introduce an assumption that is necessary to derive mathematical relations between the CTVAR, the SVAR, and the DVAR models and to provide theoretical bases for SVAR and CTVAR modeling from given time series data. Then we show a bijective correspondence between all three models. Finally, we propose a new canonical modeling approach of the objective continuous time, multivariate, linear Markov system.

The proposed modeling principles and algorithm require the following assumption.

Assumption 1. Given the CTVAR model in Equation (6) representing the objective system and a positive real constant $\Delta t>0$, which is a sampling interval of time series data for the modeling, $\sum_{m=0}^{p} S_{m} \Delta t^{p-m}$ is an invertible matrix, where $S_{p}=-I$ according to Equation (6).

As shown in the following lemma, given an objective, continuous time, multivariate, linear Markov system, this assumption always holds, if the system is stable and controllable.

LEMMA 1. Given the CTVAR model in Equation (6) representing the objective system with a positive real constant $\Delta t>0, \sum_{m=0}^{p} S_{m} \Delta t^{p-m}$ is an invertible matrix where $S_{p}=-I$, if the system is controllable and stable.

Proof. The proof is presented in Appendix A.

Assumption 1 does not apply any essential limitation to the canonical modeling of the system as long as we use the DVAR modeling, because the stability and the controllability of the objective system are required for the estimation of its valid DVAR model by using time series data observed from the system [Brockwell and Davis 1991].

To obtain the relations among the three models, first we concentrate on the canonical SVAR and CTVAR models. As we discussed in previous section, they have a bijective correspondence. However, the variables of the CTVAR model are in continuous time 
domain, while those of the SVAR model are in discrete time domain. Accordingly, we find their mathematical relations by applying a time discretization approximation to the CTVAR model. One of the most representative approximation schemes for the time discretization is the backward higher order finite difference in Equation (8), which is a natural extension of Euler formula for the $\mathrm{m}$-th derivative [Levy and Lessman 1992].

$$
Y^{(m)}(t) \simeq \frac{1}{\Delta t^{m}} \sum_{j=0}^{m}(-1)^{j} \frac{m !}{(m-j) ! j !} Y(t-j \Delta t) .
$$

This finite difference scheme is consistent, for example, the finite difference equations derived by Equation (8) converge to their original differential equation when $\Delta t \rightarrow 0$. The approximation error is $O\left(Y^{(m+1)}(t) \Delta t\right)$, which converges to zero for $\Delta t \rightarrow 0$. Moreover, the convergence of the finite difference equations' solutions provided by Equation (8) to that of the original time differential equation is ensured by Lax-Richtmyer theorem, since the objective system is linear and stable [Lax and Richtmyer 1956; Strikwerda 1989].

LEMma 2. The finite difference approximation of the CTVAR model using Equation (8) is unbiased under Assumption 1.

Proof. The proof is presented in Appendix B.

Therefore, we obtain the following lemma.

LEMMA 3. Under Assumption 1, a discrete time approximation of the CTVAR model is represented as follows.

$$
\begin{aligned}
Y(t)= & -\left(\sum_{m=0}^{p} S_{m} \Delta t^{-m}\right)^{-1} \sum_{j=1}^{p}(-1)^{j} \sum_{m=j}^{p} \frac{m !}{(m-j) ! j !} S_{m} \Delta t^{-m} Y(t-j \Delta t) \\
& -\left(\sum_{m=0}^{p} S_{m} \Delta t^{-m}\right)^{-1} W(t)
\end{aligned}
$$

and

$$
\begin{aligned}
Y(t)= & \left(I+\sum_{m=0}^{p} S_{m} \Delta t^{-m}\right) Y(t) \\
& +\sum_{j=1}^{p}(-1)^{j} \sum_{m=j}^{p} \frac{m !}{(m-j) ! j !} S_{m} \Delta t^{-m} Y(t-j \Delta t)+W(t),
\end{aligned}
$$

where $S_{p}=-I$.

Proof. The proof is presented in Appendix C.

Since the SVAR and the CTVAR models bijectively correspond to each other, Equation (10) corresponds to the SVAR model in Equation (3). Therefore, by comparing Equation (3) and Equation (10), we obtain the following representation of coefficient matrices of the SVAR in Equation (3) by coefficient matrices of the CTVAR model in Equation (6).

$$
\begin{aligned}
& \Psi_{0}=I+\sum_{m=0}^{p} S_{m} \Delta t^{-m}, \\
& \Psi_{j}=(-1)^{j} \sum_{m=j}^{p} \frac{m !}{(m-j) ! j !} S_{m} \Delta t^{-m},
\end{aligned}
$$


where $S_{p}=-I$. Thus, from a given CTVAR model, we uniquely derive a SVAR model using Equations (11) and (12).

Furthermore, we derive the coefficient matrices of the CTVAR model, $S_{m}$, from the given SVAR model by the following theorem.

THEOREM 1. Under Assumption 1, the coefficient matrices of the CTVAR model in Equation (6) are represented by coefficient matrices of the SVAR model in Equation (3) as follows.

$$
\begin{aligned}
S_{0}= & \Delta t^{-p} I \\
& -\sum_{m=1}^{p-1}\left\{(-1)^{m} \sum_{j=m}^{p-1} \frac{j !}{(j-m) ! m !} \Psi_{j}+(-1)^{p+m-1} \Delta t^{-p} \frac{p !}{(p-m) ! m !} I\right\} \\
& +(-1)^{p} \Delta t^{-p} \Psi_{p}^{-1}\left(I-\Psi_{0}\right), \\
S_{m}= & (-1)^{m} \Delta t^{m} \sum_{j=m}^{p-1} \frac{j !}{(j-m) ! m !} \Psi_{j}+(-1)^{m+p-1} \Delta t^{m-p} \frac{p !}{(p-m) ! m !} I,
\end{aligned}
$$

where $1 \leq m \leq p-1$ and $S_{p}=-I$.

Proof. The proof is presented in Appendix D.

Equations (11)-(14) indicate a bijective correspondence between the CTVAR model and its SVAR model.

We further deduce the SVAR model from the DVAR model. As was shown in Section 2.2, we need to know $Q$ or $\Psi_{0}$ for this deduction. From Theorem 1 , we derive the following Theorem 2, which provides $I-\Psi_{0}$ from the DVAR matrices only.

Theorem 2. Under Assumption 1, the matrix I- $\Psi_{0}$ is represented by the DVAR parameter matrix as follows.

$$
I-\Psi_{0}=(-1)^{p+1} \Delta t^{-p} \Phi_{p}^{-1} .
$$

Proof. The proof is presented in Appendix E.

Thus, Theorem 2 and Equations (4b) and (5b) indicate a bijective correspondence between the SVAR model and its DVAR model.

From these two theorems, we immediately provide the following corollary on the canonicality of the DVAR model.

Corollary 1. A DVAR model representing a stable, controllable, continuous time, multivariate, linear Markov system is canonical, and has a bijective correspondence with the SVAR and the CTVAR models of the system.

Under Assumption 1, the consequences provided previously enable us to obtain the SVAR model from the given DVAR model and to derive the CTVAR model from the SVAR model. Thus, we developed a novel approach, which we call CSVAR modeling [Demeshko et al. 2013]. The algorithm of the CSVAR modeling approach is shown in Figure 1. It allows us to derive both canonical models, CTVAR and SVAR, once we properly derive the DVAR model from a given time series dataset observed from a continuous time, multivariate, linear Markov system.

\section{PERFORMANCE ON ARTIFICIAL DATA}

In this section, we address objective (4) stated in Section 1. We demonstrate the performance of the proposed CSVAR modeling through numerical experiments using 


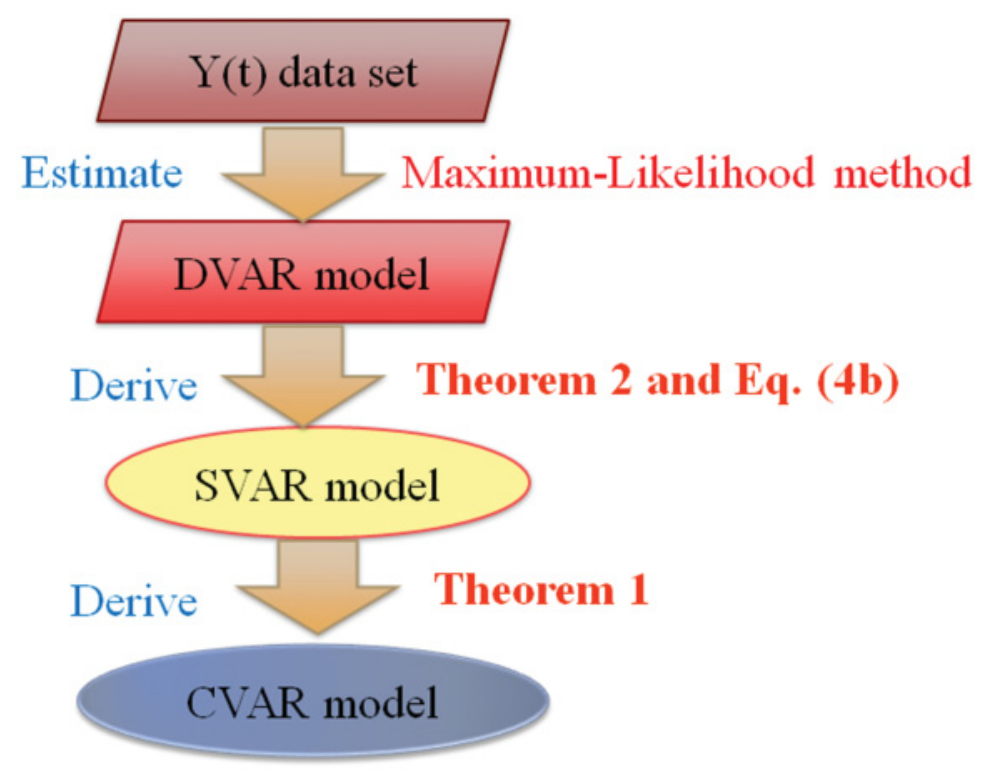

Fig. 1. The scheme of the CSVAR algorithm.

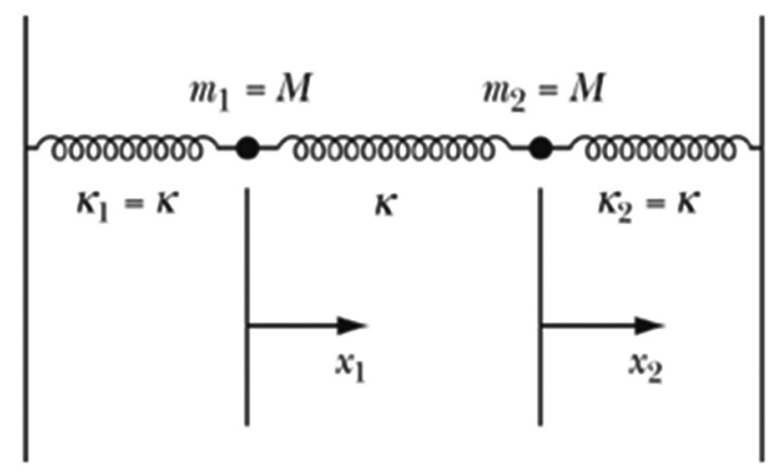

Fig. 2. Coupled oscillators.

artificial and real-world data. First, we present an illustration of the CSVAR modeling application to a simple physical system. Then we evaluate the accuracies of the canonical SVAR and CTVAR models derived from the artificial data by using our modeling method in comparison with their original models that were used to generate the data. Additionally, we confirm the applicability and the accuracy of our CSVAR approach in comparison with a past representative SVAR modeling method. Finally, we evaluate the practicality of the CSVAR approach through its application to real-world experimental data.

\subsection{Illustrative Example of CSVAR Application}

We illustrate the effectiveness of the CSVAR modeling approach through its application to a simple coupled oscillator shown in Figure 2. The dynamics of the system is 
represented by the following two differential equations.

$$
\begin{aligned}
& \ddot{x}_{1}=\frac{-2 \kappa x_{1}}{M}-\frac{c}{M} v_{1}, \\
& \ddot{x}_{2}=\frac{-2 \kappa x_{2}}{M}-\frac{c}{M} v_{2} .
\end{aligned}
$$

The mass of each object is $M$, and the spring constant of each spring is $\kappa$ where their rightmost and leftmost ends are fixed at the two walls. Two state variables $x_{1}$ and $x_{2}$ represent the deviations of the mass positions from their equilibrium, and $v_{1}=\dot{x}_{1}$ and $v_{2}=\dot{x}_{2}$ are their velocities. We also assume some damping forces acting on the masses caused by air friction with coefficient $c$.

This system is exactly represented by a controllable canonical form of the state space model in continuous time domain shown in Equation (18). This model explicitly indicates kinematics, air friction, and observation errors. It consists of the linear differential system equations having external process noises and observation equations of the state variables [Hinrichsen and Pritchard 2005].

$$
\begin{aligned}
\frac{d X}{d t} & =\left[\begin{array}{cccc}
0 & 0 & 1 & 0 \\
0 & 0 & 0 & 1 \\
\frac{-2 \kappa}{M} & \frac{\kappa}{M} & -\frac{c}{M} & 0 \\
\frac{\kappa}{M} & \frac{-2 \kappa}{M} & 0 & -\frac{c}{M}
\end{array}\right] X+\left[\begin{array}{ll}
0 & 0 \\
0 & 0 \\
1 & 0 \\
0 & 1
\end{array}\right] W \text { and } \\
Y & =\left[\begin{array}{llll}
1 & 0 & 0 & 0 \\
0 & 1 & 0 & 0
\end{array}\right]^{T} X,
\end{aligned}
$$

where $X=\left[x_{1}, x_{2}, v_{1}, v_{2}\right]^{T}, W=\left[w_{1}, w_{2}\right]^{T}$, and $w_{1}$ and $w_{1}$ are the external process noises of $x_{1}$ and $x_{2}$. The controllable canonical form of the state space model has a direct bijective correspondence to the CTVAR model [Hinrichsen and Pritchard 2005; Brockwell and Davis 1991]. Thus, for the coupled oscillator, we have the following CTVAR(2) model.

$$
Y^{(2)}(t)=\left[\begin{array}{cc}
\frac{-2 \kappa}{M} & \frac{\kappa}{M} \\
\frac{\kappa}{M} & \frac{-2 \kappa}{M}
\end{array}\right] Y^{(0)}(t)+\left[\begin{array}{cc}
-\frac{c}{M} & 0 \\
0 & -\frac{c}{M}
\end{array}\right] Y^{(1)}(t)+W(t) .
$$

We gave the values of the spring constant $\kappa=0.1 \mathrm{~N} / \mathrm{m}$, the mass $M=1 \mathrm{~kg}$, the air resistance coefficient for the mass $c=0.5 \mathrm{~N} \mathrm{~s} / \mathrm{m}$, the period of oscillation $T=2 \pi \sqrt{\frac{M}{\kappa}}=$ 19.9s. By using the CTVAR model parameters and Equation (9), we further generated a time series $Y(t)$ of 1,000 data points under a time granularity $\delta t=0.1$ s far smaller than $\Delta t$ to simulate an approximately continuous process. The external bivariate noises $W(t)$ were generated by using an i.i.d. $N\left(0, \sigma^{2}\right)$ distribution where $\sigma$ is randomly chosen from $[0.3,0.7]$ to maintain the identifiability of the CTVAR model. Under a given sampling time $\Delta t=1 \mathrm{~s}$, we further sampled the generated time series by choosing every 10 th point in it. We checked the condition of the CTVAR model with this $\Delta t$ required in Assumption 1. Moreover, because Assumption 1 is not the sufficient condition of the stability and the controllability of the system as stated in Lemma 1, these conditions of the corresponding DVAR model have been checked for the valid DVAR modeling from the generated time series. This has been performed by transforming this CTVAR model to its corresponding SVAR and DVAR models under a time granularity $\Delta t$ by using Equations (11) and (12) and the relation of Equation (4a). The stability of the DVAR model was assessed by checking whether all eigenvalues of the system matrix $A_{D}$ in the 
Table I. The Parameter Matrices of the Original and Estimated DVAR, SVAR, and CTVAR Models, where $Z$ and $Z$ Represent Original and Estimated Matrices, Respectively, for $Z=\Phi, \Psi$, and $S$

\begin{tabular}{|c|c|c|c|c|c|c|c|c|}
\hline \multicolumn{3}{|c|}{ DVAR model } & \multicolumn{3}{|c|}{ SVAR model } & \multicolumn{3}{|c|}{ CTVAR model } \\
\hline \multirow{2}{*}{$\Phi_{1}$} & 1.476 & 0.087 & \multirow{2}{*}{$\Psi_{0}$} & -0.700 & 0.100 & \multirow{2}{*}{$S_{0}$} & -0.200 & 0.100 \\
\hline & 0.087 & 1.476 & & 0.100 & -0.700 & & 0.100 & -0.200 \\
\hline \multirow{2}{*}{$\hat{\Phi}_{1}$} & 1.471 & 0.088 & \multirow{2}{*}{$\hat{\Psi}_{0}$} & -0.708 & 0.105 & \multirow{2}{*}{$\hat{S}_{0}$} & -0.205 & 0.101 \\
\hline & 0.088 & 1.473 & & 0.102 & -0.702 & & 0.101 & -0.204 \\
\hline \multirow{2}{*}{$\Phi_{2}$} & $\mid-0.590$ & -0.035 & \multirow{2}{*}{$\Psi_{1}$} & 2.50 & 0 & \multirow{2}{*}{$S_{1}$} & -0.5 & 0 \\
\hline & -0.035 & -0.590 & & 0 & 2.50 & & 0 & -0.5 \\
\hline \multirow{2}{*}{$\hat{\Phi}_{2}$} & -0.588 & -0.036 & \multirow{2}{*}{$\hat{\Psi}_{1}$} & 2.503 & -0.004 & \multirow{2}{*}{$\hat{S}_{1}$} & -0.503 & 0.004 \\
\hline & -0.035 & -0.590 & & -0.001 & 2.498 & & 0.001 & -0.498 \\
\hline & & & \multirow{2}{*}{$\Psi_{2}$} & -0.67 & 0 & & & \\
\hline & & & & 0 & -0.67 & & & \\
\hline & & & \multirow{2}{*}{$\hat{\Psi}_{2}$} & -1.00 & 0 & & & \\
\hline & & & & 0 & -1.00 & & & \\
\hline
\end{tabular}

state space model corresponding to the DVAR model in the discrete time domain have their absolute values less than 1 [Brockwell and Davis 1991; Stamer et al. 1996]. We checked the controllability conditions by confirming if $\left[B_{D} A_{D} B_{D} \ldots A_{D}^{3} B_{D}\right]$ is row full rank, where $B_{D}$ is the noise matrix in the state space model [Brockwell and Davis 1991].

Finally, we applied the CSVAR algorithm shown in Figure 1 to this sampled time series and estimated the DVAR, SVAR, and CTVAR models, using provided correct model order, $p=2$. Table I shows the comparison between the original models derived from the CTVAR model and the estimated models. We see that the SVAR and CTVAR models estimated by the CSVAR approach and their original models match well. We also note that the original DVAR model matches well with the DVAR model estimated by the Maximum-Likelihood method. The last corresponds to Corollary 1, showing that the objective system is represented by a unique DVAR model when it is a linear Markov, stable, controllable, and observable system in continuous time domain. Accordingly, we see that the CSVAR modeling appropriately reconstructs the original SVAR and CTVAR models of the system from a given time series in this example. These models provide the valid canonical relations between the variables in the original system.

\subsection{Accuracy of the Proposed Method}

We demonstrate the accuracy of the SVAR and the CTVAR models derived by the CSVAR modeling. For this purpose, we perform a set of computer simulations. The procedure of the numerical experiments is similar to the one described in the illustrative example, and it is as follows.

(1) We artificially generate parameter matrices of the CTVAR model, $S_{m}(j=0, \ldots, p-$ 1 ), each element of which is generated by a uniformly distributed random value in the interval $(-1.5,1.5)$.

(2) Then we generate a CTVAR time series data $Y(t)$ by using Equation (9) under a time granularity $\delta t=0.1 \Delta t$ to approximately simulate a continuous process. We also generate a multivariate i.i.d. Gaussian time series $W(t)$. The mean value of each element in $W(t)$ is set to be zero, and its standard deviation is randomly chosen from $[0.3,0.7]$ to maintain the identifiability of the CTVAR model. We check if this CTVAR model satisfies our Assumption 1. If not, we repeat the process from (1) to (2).

(3) We transformed this CTVAR model to its corresponding SVAR and DVAR models by using Equations (11) and (12) and Equation (4a), respectively, under a time granularity $\Delta t$. Then, we check the conditions of the stability and controllability of the transformed DVAR model similarly to the illustrative example in Section 4.1. 


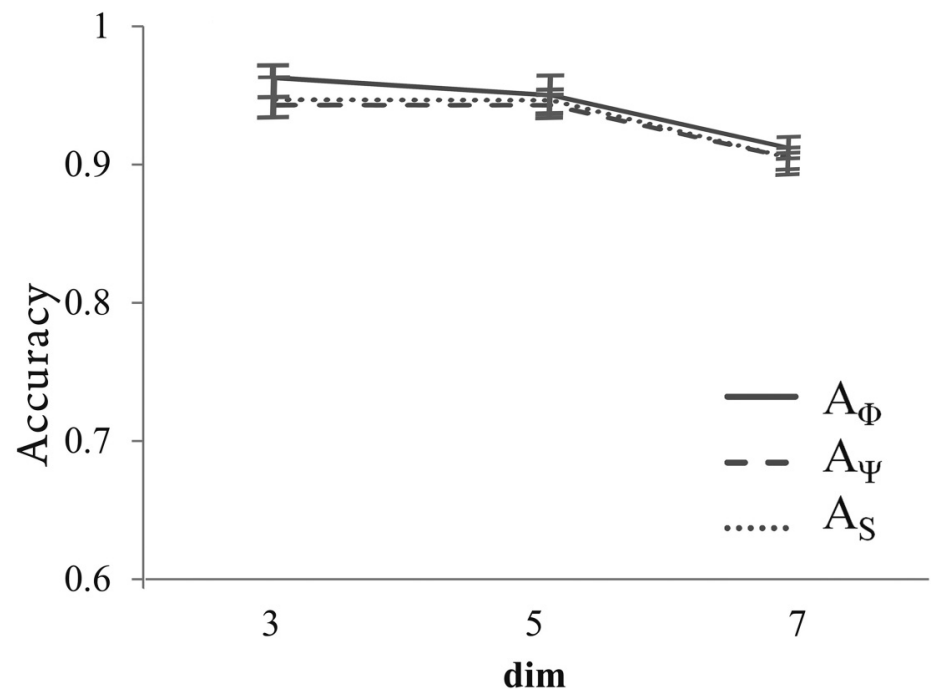

Fig. 3. Accuracy over different dimensions $d$, when $p=2, N=1,000$, and $\Delta t=1$ s.

If they are not held, we repeat the process from (1) to (3), till we obtain parameters for the stable and controllable model.

(4) We estimate the $\mathrm{AR}$ matrices, $\Phi_{j}(j=1, \ldots, p)$, as $\hat{\Phi}_{j}(j=1, \ldots, p)$ from the generated multivariate time series $Y(t)$ by a representative DVAR modeling algorithm. Here, we use the Maximum Likelihood method to derive AR parameter matrices [Shea 1987].

(5) We estimate the SVAR matrices, $\Psi_{j}(j=0, \ldots, p)$, as $\hat{\Psi}_{j}(j=0, \ldots, p)$ by Equation (4b) and Equation (15). Subsequently, we estimate the CTVAR matrices, $S_{m}(j=0, \ldots, p-1)$, as $\hat{S}_{m}(j=0, \ldots, p-1)$ by Equation (13) and Equation (14).

(6) We evaluate the accuracy of the estimated matrices over the original matrices, using the following cosine measure that represents an elementwise accuracy averaged over all matrices in a model.

$$
A_{x}=\frac{1}{p} \sum_{k=1}^{p} \frac{\sum_{i j} \hat{x}_{k, i j} x_{k, i j}}{\sqrt{\sum_{i j} \hat{x}_{k, i j}^{2}} \sqrt{\sum_{i j} x_{k, i j}^{2}}},
$$

where $x_{k, i j}$ is the $i, j$-element of an original matrix $X_{k}$, which is the AR matrix of the $k$-th order, and $\hat{x}_{k, i j}$ is the $i, j$-element of an original matrix $\hat{X}_{k}$, which is an estimation of $X_{k}$. The summation $\sum_{i j}$ is taken over all elements in $X_{k}$ and $\hat{X}_{k}$, respectively. Thus, Equation (19) represents an elementwise accuracy averaged over all AR matrices in a model. We apply it to evaluate the accuracy of $X_{k}=\Phi_{j}$, $\Psi_{j}$ or $S_{m}$ where $k=j$ or $m$.

We chose a default parameter setting of the dimension of $Y(t), d=5$, the number of timesteps of $Y(t), N=1,000$, the order of the CTVAR model, $p=2$, and time granularity $\Delta t=1 \mathrm{~s}$, for data generation. Then, we assessed the estimation accuracy over various values of each parameter while setting the other parameters to their default values. For every parameter setting, we repeated 20 experiments and evaluated their 20 accuracies $A_{x}$ for each experiment.

Figures 3, 4, 5, and 6 show the comparisons of the estimation accuracy over the various values of every parameter. In Figure 3 , the lines show the accuracies of AR matrix estimations averaged over the 20 experiments for the DVAR, the SVAR, and 


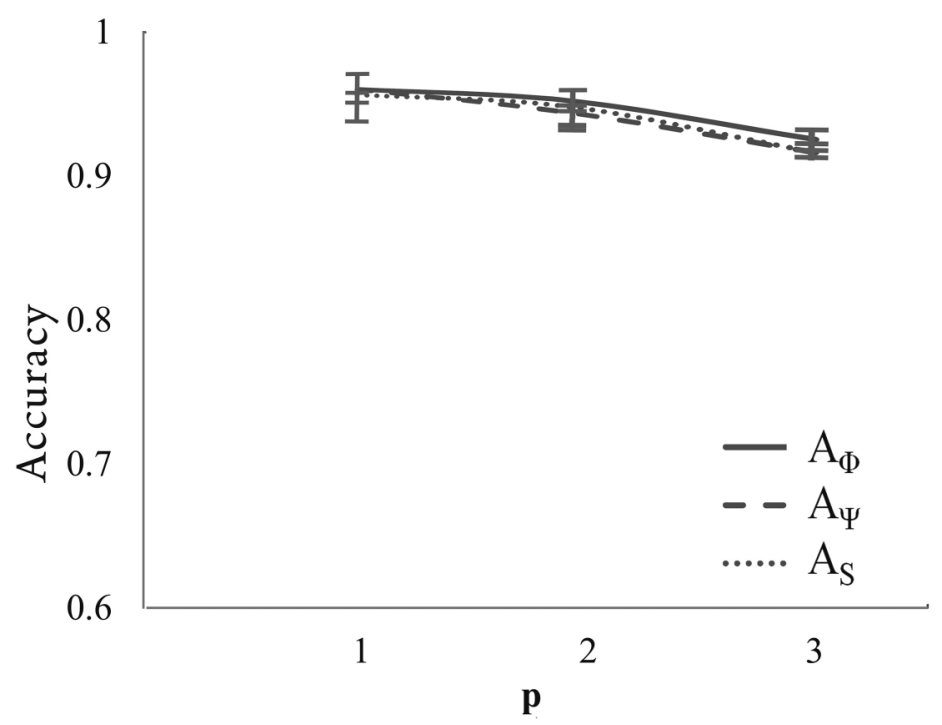

Fig. 4. Accuracy over different AR orders $\mathrm{p}$, when $\mathrm{d}=5, \mathrm{~N}=1,000$ and $\Delta t=1 \mathrm{~s}$.

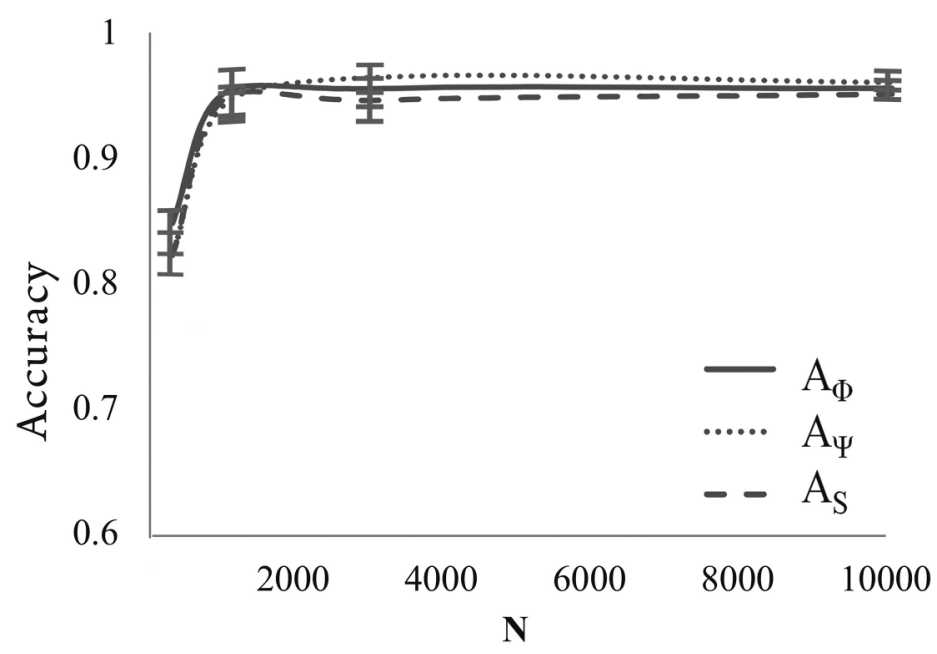

Fig. 5. Accuracy over different steps $N$, when $d=5, p=2$, and $\Delta t=1 \mathrm{~s}$.

the CTVAR models, respectively, under multiple dimensions of $Y(t), d=3,5$, and 7 , where the error bars represent the standard deviations. We observe the high accuracies of the SVAR and the CTVAR matrices, $A_{\Psi}$ and $A_{S}$, evaluated by the proposed CSVAR approach as well as the ones of the DVAR matrices, $A_{\Phi}$, obtained from the dataset for all dimensions of the observation vectors. However, for the larger dimension $d$ the accuracy slightly degrades. This was expected, since the number of parameters to be estimated in the AR matrices is $O\left(d^{2}\right)$ and many parameters under a large $d$ make the estimation of the DVAR model statistically unstable for the same length of the given time series data. In Figure 4, we see the results for different AR orders, $p=1,2$, and 3. Here, we also note that all three estimated models have high accuracies in all cases. However, similarly to the cases of the large $d$, the model estimation of the higher orders $p$ shows some degraded accuracies, since the model becomes more complex with 


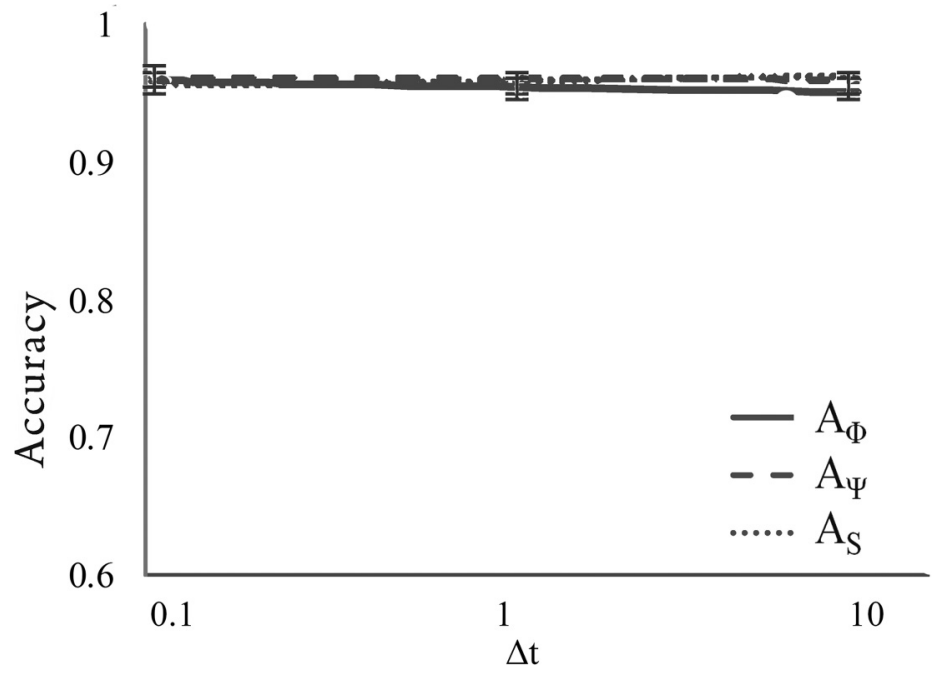

Fig. 6. Accuracy over different $\Delta t$, when $d=5, p=2$, and $N=1,000$.

more parameters to be estimated. Figure 5 shows the results for the datasets with the different number of steps, $N=300,1,000,3,000$, and 10,000. Here, we observe limited accuracy in the small sample case, $N=300$. This is easily explained by the statistical instability. Under the larger $N$, the accuracies of all three models estimations are significantly high. Finally, in Figure 6, we see the results for different time granularity $\Delta t=0.1,1$, and 10 . We see that overall results do not depend on parameter $\Delta t$ as far as the conditions of Assumption 1; the stability and the controllability of the DVAR model hold.

In summary, our proposed CSVAR modeling accurately captures the system dynamics and the dependency structure among its variables in forms of the SVAR and the CTVAR models.

\subsection{Comparison of CSVAR with AR-LiNGAM}

In this subsection, we compare our proposed CSVAR modeling with a past representative method for deriving the SVAR model and evaluate its applicability under various conditions. As was shown in Section 2.1, most of the SVAR modeling approaches [Gottschalk 2001; Pfaff and Kronberg 2008; Kilian 2011] require some strong prior knowledge, which makes them incomparable with the CSVAR approach. However, the AR-LiNGAM method [Hyvarinen et al. 2008] requires weaker assumptions on the system such as acyclic $\Psi_{0}$ and non-Gaussian noises. Therefore, we concentrate on the comparisons with AR-LiNGAM. The AR-LiNGAM approach derives the external noise time series, $U(t)$, by the maximum-likelihood estimation of the DVAR model; then it obtains ordering information of variables in $Y(t)$ by applying ICA. This ordering information together with the orthonormality of the noises further provides matrix $\Psi_{0}$ of the SVAR model.

We compared the CSVAR and the AR-LiNGAM approaches by generating four sets of artificial data: (a) non-Gaussian and acyclic case, (b) Gaussian and acyclic case, (c) nonGaussian case without the acyclicity assumption, and (d) Gaussian case without the acyclicity assumption. To generate non-Gaussian noise, we independently draw the noise values in $W(t)$ from Gaussian distributions and subsequently pass them through a power nonlinearity (raising the absolute value to an exponent in the interval [0.5, 0.8 ] or $[1.2,2.0]$, but keeping the original sign) to make them non-Gaussian [Shimizu et al. 2011]. To generate Gaussian noise, we use the identical process with that of 


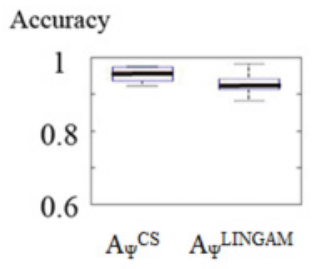

(a)

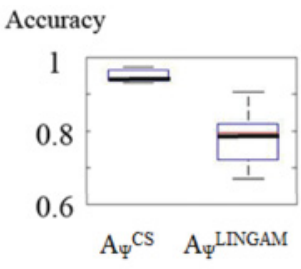

(b)

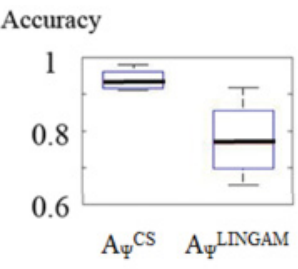

(c)

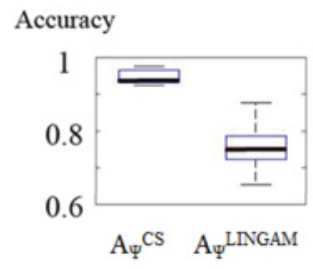

(d)

Fig. 7. Accuracies of the SVAR model estimations by CSVAR and AR-LINGAM for different processes under $d=5, N=1,000$, and $p=2$.

the previous subsection. To generate the acyclic case, we produce SVAR parameter matrices, where matrix $\Psi_{0}$ is strictly lower triangular for the necessary condition of acyclicity. Then, we check regularity of the matrix $\Phi_{p}$ for Equation (13) to ensure the existence of a CTVAR model corresponding to the generated SVAR. If the CTVAR model exists, we continue the model generation process in the same way as described in the previous subsection. To generate the case without the acyclicity assumption, we do not limit $\Psi_{0}$ to a strictly lower-triangular matrix.

Thereafter, we apply both our proposed CSVAR and the AR-LiNGAM to the generated datasets. Both approaches give us SVAR model parameter matrices $\Psi_{j}^{C S}(j=0, \ldots, p)$ and $\Psi_{j}^{L i N G A M}(j=0, \ldots, p)$. To evaluate the accuracies of the estimated matrices, we compare them with the original ones by Equation (19). In Figure 7(a), we see the box plots of the accuracies of the CSVAR and the AR-LiNGAM approaches where a bold line is the median, lower and higher edges of a box are the 25th and 75th percentiles, and the length of a whisker represents the lowest datum within $1.5 \mathrm{IQR}$ (Interquartile Range) of the lower quartile and the highest datum within $1.5 \mathrm{IQR}$ of the upper quartile. Through these comparisons, we see that both modeling approaches show a very good performance in the non-Gaussian and acyclic case. In Figures 7(b), 7(c), and 7(d), we see that the accuracies of the AR-LiNGAM are substantially lower than those of our CSVAR method, in the cases when the assumptions of non-Gaussianity and acyclicity are not met. Accordingly, the applicability of the AR-LiNGAM approach is limited to the non-Gaussian and acyclic cases, while our proposed CSVAR modeling is widely applicable to the continuous time linear Markov system as far as the system is stable and controllable.

\subsection{Performance Evaluation by Using Real-World Data}

In this subsection, we present the application of the CSVAR approach to a real-world experimental data to evaluate its practicality. In our study, we used reactor noise time series measured in an impulse fast neutron research reactor named IBR-2 at Joint Institute of Nuclear Research in Dubna, Russia [Pepyolyshev 1988]. This reactor has a unique structure (Figure 8). It uses rotating main and additional neutron reflectors driven by motors for power pulse initiation. They reflect the generated neutrons back to the core, when they approach the reactor core. This increases the number of neutrons and activates the fission chain process in the reactor core. That occurs in a very short period and produces power pulses, since both reflectors rotate very fast. Also, according to specifications of the reactor design, the effect of the neutron reflection of the main neutron reflector is almost four times bigger than that of the additional neutron reflector.

We analyzed the time series of the peak values of the power pulses, $Q$, axial deviations of the main neutron reflector, $X_{Q}$, and of the additional neutron reflector, $X_{A}$, measured during the stable reactor operation. The axial deviations of the reflectors 


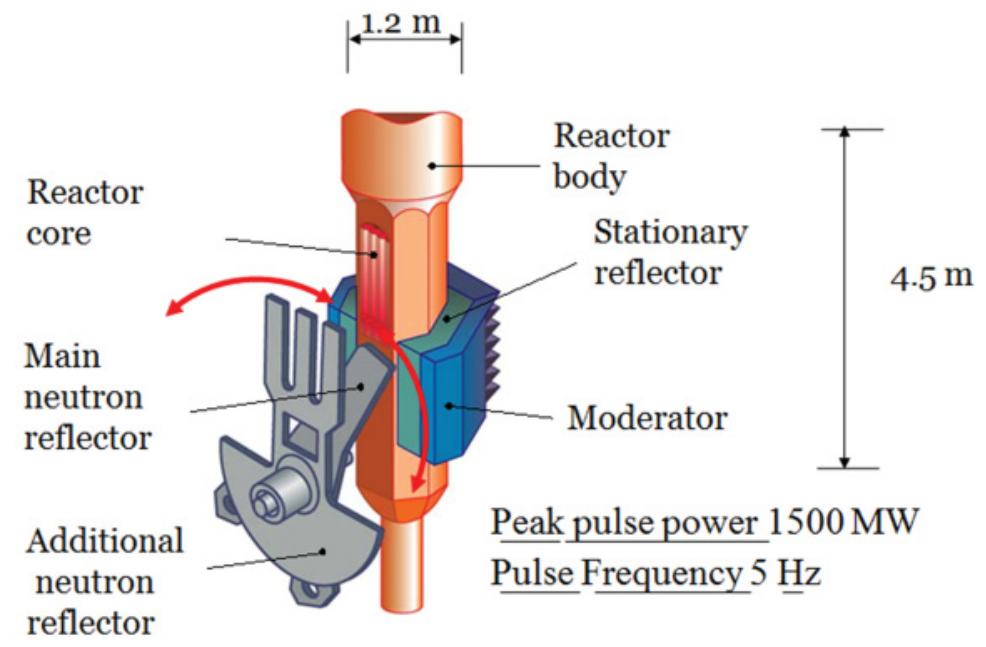

Fig. 8. The scheme of IBR-2 impulse fast neutron reactor.

are their angular deviation from the vertical central line of the reactor core. This time series contains 8,192 timestep measurements. Every variable of the dataset has been normalized to give a zero mean and a unit standard deviation. Sampling frequency of the time series data is equal to the frequency of the pulse operation of IBR-2, which is $5 \mathrm{~Hz}$ [Pepyolyshev 1988].

It is known that the relations between the heat removal from the core and the negative feedback effect of core temperature to power generation is approximately represented by the second order delay process. Also, the sinusoid impact of periodically rotating reflectors is approximated by the second order delay process. Accordingly, the dynamics of this reactor can be approximated by DVAR(2). After the DVAR(2) estimation by the Maximum-Likelihood method, we further calculated its SVAR and CTVAR parameters using the CSVAR approach. The results are presented in Equations (20)-(26).

Equation (20) shows the parameter matrix $\Psi_{0}$ of the SVAR model that represents the fast effects among variables, that is, the effects that propagate within less than a sampling period. We see the significant values of the $(1,2)$ and $(1,3)$ elements of the matrix, which correspond to the influence of the main neutron reflector's axial deviation, $X_{Q}$, on the peak values of the power pulses, $Q$, and to the influence of $X_{A}$ on $Q$, respectively. Also, the ratio of these two numbers is close to four, which coincides with the ratio of both reflectors' efficiency on the neutron reflection. Further, we see that the other elements of $\Psi_{0}$ are relatively small, which means that there is no impact from the power output to deviations of both neutron reflectors, and no influence between the reflectors. This result corresponds to the system dynamics of IBR-2 reactor, where both reflectors initiate the power pulse and the rotation of the reflectors is independently driven by motors, that is, it does not depend on the reactor power.

$$
\Psi_{0}=\left[\begin{array}{rrr}
Q & X_{Q} & \multicolumn{1}{c}{X_{A}} \\
1.09 & 11.79 & 2.72 \\
0.60 & -0.86 & 0.10 \\
-0.53 & 0.53 & -1.11
\end{array}\right] \underset{X_{Q}}{X_{A}}
$$

Equation (21) shows the matrix $\Psi_{1}$ of the SVAR model, which represents the delayed effects among the variables. We see, that $\Psi_{1}$ has a structure similar to $\Psi_{0}$. This implies that the impacts of the two neutron reflectors are also significant in the first order delay 
effect. This result is also consistent with the aforementioned physical background of the IBR-2 reactor.

$$
\Psi_{1}=\left[\begin{array}{crc}
Q & X_{Q} & \multicolumn{1}{c}{X_{A}} \\
3.11 & 90.65 & 18.91 \\
0.58 & 1.93 & -4.49 \\
2.80 & -1.51 & -1.78
\end{array}\right] \begin{aligned}
& Q \\
& X_{Q} \\
& X_{A}
\end{aligned}
$$

We note, that $\Psi_{2}$ in Equation (22) is diagonal. That well corresponds to Equation (12), from which we have $\Psi_{p}=(1)^{p} \Delta t^{-p} S_{p}$ and $S_{p}=I$. Therefore, matrix $\Psi_{2}$ reflects the fact that we assume the objective system to be a continuous time, linear Markov system represented by a CTVAR process in our CSVAR modeling.

$$
\Psi_{2}=\left[\begin{array}{rrr}
Q & X_{Q} & \multicolumn{1}{c}{X_{A}} \\
-25.00 & 0.00 & 0.00 \\
0.00 & -25.00 & 0.00 \\
0.00 & 0.00 & -25.00
\end{array}\right] \begin{gathered}
Q \\
X_{Q} \\
X_{A}
\end{gathered}
$$

If we look at the DVAR parameter matrices $\Phi_{1}$ and $\Phi_{2}$ that represent the delayed effects between the variables, we see that they are not consistent with the dynamics of the IBR-2 reactor. The matrix $\Phi_{1}$ presented in Equation (23) does not show very significant magnitudes of the $(1,2)$ and $(1,3)$ elements in comparison with the other elements. In addition, their values are negative, while the impacts of the neutron reflectors to the power should be positive. The matrix $\Phi_{2}$ in Equation (24) does not show very clear structure, either. Such inconsistency occurs, since the fast and the delayed effects are not decomposed in the DVAR model.

$$
\begin{gathered}
\Phi_{1}=\left[\begin{array}{crr}
Q & X_{Q} & \multicolumn{1}{c}{X_{A}} \\
-0.021 & -1.689 & -0.492 \\
-0.007 & -0.118 & 0.066 \\
-0.059 & -0.158 & -0.031
\end{array}\right] \begin{array}{c}
Q \\
X_{Q}, \\
X_{A}
\end{array} \\
\Phi_{2}=\left[\begin{array}{rrr}
0.476 & -0.654 & -0.203 \\
0.021 & 0.481 & -0.062 \\
0.055 & -0.138 & 0.496
\end{array}\right] \begin{array}{c}
Q \\
X_{Q} . \\
X_{A}
\end{array}
\end{gathered}
$$

The proposed CSVAR approach also provides the CTVAR model of the nuclear reactor system. This model is canonical and gives us the information on relations among the reactor's processes in continuous time domain. The structure of the CTVAR parameter matrices presented in Equation (25) and Equation (26),

$$
\begin{aligned}
& \begin{array}{lll}
Q & X_{Q} & X_{A}
\end{array} \\
& S_{0}=\left[\begin{array}{rrr}
24.27 & 161.46 & 46.64 \\
-2.21 & 26.60 & 0.57 \\
-3.06 & 4.50 & 21.93
\end{array}\right] \begin{array}{c}
Q \\
X_{Q} \\
X_{A}
\end{array} \\
& S_{1}=\left[\begin{array}{rrr}
Q & X_{Q} & X_{A} \\
9.38 & -18.13 & -3.78 \\
-0.12 & 9.61 & 0.90 \\
-0.56 & 0.30 & 10.36
\end{array}\right] \begin{array}{c}
Q \\
X_{Q} \\
X_{A}
\end{array},
\end{aligned}
$$

is similar to that of the SVAR matrices. Both $S_{0}$ and $S_{1}$ have significant values in $(1,2)$ and $(1,3)$ elements, whose ratio is close to four. However, these elements are negative in $S_{1}$, because of the negative feedback of the peak power. This effect occurs as follows. Once the neutron population is increased in the core by the reactor, the core 
temperature is increased through the activation of the nuclear fission chain reaction. The increase of the temperature reduces the efficiency of the individual nuclear fission reaction, and this suppresses the power generation. These feedback processes are reflected by the negative signs of the main reflectors' impacts in $S_{1}$.

In summary, the application of our CSVAR modeling gives us information on the dependency structure of nuclear reactor processes. Particularly, it presents the fast influences that propagate in the system during a sampling period. The last became possible through the mathematical reconstruction of the SVAR and the CTVAR models out of the DVAR model.

\section{DISCUSSIONS AND CONCLUSION}

The CSVAR modeling approach presented in this study belongs to the framework of canonical modeling of multivariate, linear Markov systems. There are few studies on the canonical and/or causal modeling of the dynamic Markov systems from given time series data in the statistical causal inference [Moneta et al. 2010; Hyvarinen et al. 2008; Gottschalk 2001; Pfaff and Kronberg 2008; Kilian 2011]. The advantage of the CSVAR approach is that CSVAR is based on a very generic assumption and does not require any specific domain knowledge. In this sense, the CSVAR approach is more comparable with the study by Voortman et al. [2010], which proposed a method to learn causal structures of the continuous time Markov systems in the framework of the statistical causal inference. Though its basic framework and objective is different from our proposed method, it shares some similar features with ours such that its model consists of higher order time difference variables and requires faithfulness of the objective system ensured by excluding its equilibrium states. The latter feature seems to be associated with the requirement of the controllability in our approach, since some parts of the system can stay at the equilibrium unless the system is fully controllable by the random noises.

In this study, we achieved all four objectives. First, we showed that the DVAR model is canonical upon an assumption on the objective continuous time, multivariate, linear Markov system. Second, we discovered mathematical relations between the CTVAR and the SVAR models and the DVAR model of the system. Third, by applying our proposed CSVAR modeling, we accurately derived a canonical representation of an objective system behind a given time series dataset under a generic assumption that does not limit its applicability when the system is well approximated by a continuous time, multivariate, linear Markov system. Finally, we demonstrated the applicability and the accuracy of the CSVAR modeling through some numerical experiments using both artificial and real-world data, where it showed a good performance.

We conclude that the CSVAR modeling provides a highly generic methodology to empirically derive a unique model that reflects some elementary rules governing the objective system. This methodology is applicable in many fields, for example, reactor noise analysis, economics, bioinformatics, and so on. The canonical models derived by the CSVAR approach can be used to analyze the observed system, even when background knowledge is limited and its inside processes are unknown. The CTVAR and the SVAR models provided by this approach bijectively correspond to the objective system dynamics and give us the important information on the system's structural change. Hence, our CSVAR approach enables us to empirically derive scientific models and their associated laws in the system. Such models can be used to monitor and diagnose anomalies of an objective system through time series measurements. For example, in the case of the IBR-2 reactor presented in Section 6, if we observe some anomalous changes at $(1,2)$ and $(1,3)$ elements of the CTVAR and the SVAR matrices, we can infer that defects of the neutron reflectors and/or the neutron generation process are occurring. Furthermore, the models derived by our approach can help us to improve or discover new knowledge on the system. 


\section{A. THE PROOF OF LEMMA 2}

Since the CTVAR model in Equation (6) bijectively corresponds to the dynamics of a stable, controllable, multivariate, linear Markov system it can be represented by a controllable canonical form of its state space model [Brockwell and Davis 1991; Stamer et al. 1996] as follows.

$$
\begin{aligned}
d X_{c}(t) / d t & =A_{c} X_{c}(t)+B_{c} W(t), \\
Y(t) & =C_{c}^{T} X_{c}(t),
\end{aligned}
$$

where $X_{c}(t)$ is a $d p$-dimensional state variable vector $X_{c}(t)=\left[x_{c}^{(0)}(t)^{T} \ldots x_{c}^{(p-1)}(t)^{T}\right]^{T}$, which is a concatenation of the $m$-th time derivative of a $d$-dimensional state variable vector $x_{c}(t)\left(=x_{c}^{(0)}(t)\right), m=0, \ldots, p-1$. The $d p \times d p$ matrix $A_{c}$, and $d p \times d$ matrices $B_{c}$ and $C_{c}$ are given as follows.

$$
\begin{gathered}
A_{c}=\left[\begin{array}{ccccc}
0 & I & 0 & \ldots & 0 \\
0 & 0 & I & \ldots & 0 \\
\vdots & \vdots & \vdots & \ddots & \vdots \\
0 & 0 & 0 & \ldots & I \\
S_{0} & S_{1} & S_{2} & \ldots & S_{p-1}
\end{array}\right], \\
B_{c}=\left[\begin{array}{lllll}
0 & 0 & \ldots & 0 & I
\end{array}\right]^{T} \text { and } C_{c}=\left[\begin{array}{llll}
I & 0 & \ldots & 0
\end{array}\right]^{T},
\end{gathered}
$$

where $S_{m}, m=0, \ldots, p-1$ are given in Equation (6). When the system is stable, all eigenvalues of the system matrix in the state space model, that is, the solutions $z$ of $\operatorname{det}\left|\sum_{m=0}^{p} S_{m} z^{-m}\right|=0$, have negative real parts [Brockwell and Davis 1991; Stamer et al. 1996]. This implies that $\operatorname{det}\left|\sum_{m=0}^{p} S_{m} \Delta t^{-m}\right| \neq 0$, since $\Delta t>0$. Thus, $\sum_{m=0}^{p} S_{m} \Delta t^{-m}$ is an invertible matrix.

\section{B. THE PROOF OF LEMMA 2}

Proof. If the mean of $W^{(2)}(t)$ over time, $E_{t}\left[W^{(2)}(t)\right]$, is nonzero, $W(t)$ mean value changes over time, then it is not stationary, hence, not stable. This is contradictory to Assumption 1. Thus, $E_{t}\left[W^{(2)}(t)\right]=0$. Take the second time derivatives of Equation (27) as

$$
\begin{aligned}
X_{c}^{(3)}(t) & =A_{c} X_{c}^{(2)}(t) / d t+B_{c} W(t)^{(2)}(t), \\
Y^{(2)}(t) & =C_{c}^{T} X_{c}^{(2)}(t) .
\end{aligned}
$$

$X_{c}^{(2)}(t)$ is analytically solved as follows.

$$
X_{c}^{(2)}(t)=\exp \left(A_{c} t\right) X_{c}^{(2)}(0)+\int_{0}^{t} \exp \left(A_{c} \tau\right) B_{c} W^{(2)}(\tau) d \tau .
$$

Because this system is stable,

$$
\begin{aligned}
E_{t}\left[Y^{(2)}(t)\right] & =C_{c}^{T} E_{t}\left[X_{c}^{(2)}(t)\right]=C_{c}^{T} E_{t}\left[\int_{0}^{t} \exp \left(A_{c} \tau\right) B_{c} W^{(2)}(\tau) d \tau\right] \\
& =C_{c}^{T} \int_{0}^{t} \exp \left(A_{c} \tau\right) B_{c} E_{t}\left[W^{(2)}(\tau)\right] d \tau=0 .
\end{aligned}
$$

Similarly, $E_{t}\left[Y^{(m+1)}(t)\right]=0$ for all $m=1, \ldots, p$. 
The application of the backward higher order finite difference, Equation (8), to each time derivative term in the CTVAR model, Equation (6), has the following approximation error $\operatorname{Err}(t)$, since its application to $F^{(m)}(t)$ has the error of $O\left(F^{(m+1)}(t) \Delta t\right)$.

$$
\operatorname{Err}(t)=\sum_{m=1}^{p} \alpha_{m} Y^{(m+1)}(t) \Delta t+\sum_{m=1}^{q} \beta_{m} W^{(m+1)}(t) \Delta t,
$$

where $\alpha_{m}$ and $\beta_{m}$ are nonzero constants. Accordingly, the following holds.

$$
E_{t}[\operatorname{Err}(t)]=\sum_{m=1}^{p} \alpha_{m} E_{t}\left[Y^{(m+1)}(t)\right] \Delta t+\sum_{m=1}^{q} \beta_{m} E_{t}\left[W^{(m+1)}(t)\right] \Delta t=0 .
$$

Thus, the approximation error is unbiased.

\section{THE PROOF OF LEMMA 3}

By substituting Equation (27) into Equation (6), we obtain the following.

$$
\begin{aligned}
& \frac{1}{\Delta t^{p}} \sum_{j=0}^{p}(-1)^{j} \frac{p !}{(p-j) ! j !} Y(t-j \Delta t) \\
& \quad=\sum_{m=0}^{p-1} S_{m} \frac{1}{\Delta t^{m}} \sum_{j=0}^{m}(-1)^{j} \frac{m !}{(m-j) ! j !} Y(t-j \Delta t)+W(t) .
\end{aligned}
$$

The terms of $Y(t-j \Delta t)$ are summarized with $S_{p}=-I$ and rewritten by permuting the summations on $m$ and $j$.

$$
\begin{aligned}
& \sum_{m=0}^{p} S_{m} \Delta t^{-m} \sum_{j=0}^{m}(-1)^{j} \frac{m !}{(m-j) ! j !} Y(t-j \Delta t) \\
& \quad=\sum_{j=0}^{p}(-1)^{j} \sum_{m=j}^{p} \frac{m !}{(m-j) ! j !} S_{m} \Delta t^{-m} Y(t-j \Delta t) .
\end{aligned}
$$

By substituting these relationships into Equation (28), we obtain the following.

$$
-\sum_{m=0}^{p} S_{m} \Delta t^{-m} Y(t)=\sum_{j=1}^{p}(-1)^{j} \sum_{m=j}^{p} \frac{m !}{(m-j) ! j !} S_{m} \Delta t^{-m} Y(t-j \Delta t),
$$

where $S_{p}=-I$ and $R_{0}=I$. By Assumption 1 and Lemma $1, \sum_{m=0}^{p} S_{m} \Delta t^{-m}$ is an invertible matrix. Then, by multiplying both sides of the equation by $-\left(\sum_{m=0}^{p} S_{m} \Delta t^{-m}\right)^{-1}$, we obtain Equation (19).

\section{THE PROOF OF THEOREM 1}

Equation (12) becomes as follows in the case of $j=p-1$ with $S_{p}=-I$ defined in Assumption 1.

$$
\begin{array}{ll} 
& \Psi_{p-1}=(-1)^{p-1} \Delta t^{-p+1} S_{p-1}-(-1)^{p-1} \Delta t^{-p} \frac{p !}{(p-1) ! 1 !} I . \\
\text { Therefore, } & S_{p-1} \Delta t^{-p+1}=(-1)^{p-1} \Psi_{p-1}+\Delta t^{-p} p I .
\end{array}
$$


Let us assume that Equation (14) is an expression for general case $1 \leq m \leq p-1$; we rewrite it as follows.

$$
S_{m} \Delta t^{-m}=(-1)^{m} \sum_{j=m}^{p-1} \frac{j !}{(j-m) ! m !} \Psi_{j}+(-1)^{m+p-1} \Delta t^{-p} \frac{p !}{(p-m) ! m !} I .
$$

Note that Equation (30) subsumes Equation (29). If we write Equation (12) for the case $j=p$, we get

$$
\Psi_{p}=(-1)^{p-1} \Delta t^{-p} I
$$

Then we rewrite Equation (30) as follows.

$$
S_{m} \Delta t^{-m}=(-1)^{m} \sum_{j=m}^{p} \frac{j !}{(j-m) ! m !} \Psi_{j}
$$

We rewrite Equation (12) for the case $j=k-1$ by substituting Equation (32) as follows.

$$
\Psi_{k-1}=(-1)^{k-1} S_{k-1} \Delta t^{-(k-1)}+(-1)^{k-1} \sum_{m=k}^{p} \frac{m !}{(m-(k-1)) !(k-1) !}(-1)^{m} \sum_{j=m}^{p} \frac{j !}{(j-m) ! m !} \Psi_{j}
$$

By changing the order of the double summation in the last term,

$$
(-1)^{k-1} S_{k-1} \Delta t^{-(k-1)}=\Psi_{k-1}-(-1)^{k-1} \sum_{j=k}^{p} \Psi_{j} \sum_{m=k}^{j}(-1)^{m} \frac{m !}{(m-(k-1)) !(k-1) !} \frac{j !}{(j-m) ! m !} I
$$

is obtained. Then, we further obtain the following expression.

$$
\begin{aligned}
& (-1)^{k-1} S_{k-1} \Delta t^{-(k-1)} \\
& =\Psi_{k-1}-\sum_{j=k}^{p} \Psi_{j} \frac{j !}{(k-1) !(j-k+1) !}\left(\sum_{u=0}^{j-k+1}(-1)^{u} \frac{(j-k+1) !}{(u) !(j-u-k+1) !} I-I\right),
\end{aligned}
$$

where $m=u+k-1$. The summation over $u$ is zero based on binomial theorem, and further rewriting $k-1$ in the formula by $m-1$, we know that Equation (32) holds for $m-1$. By induction, Equation (32) holds for $1 \leq m \leq p-1$. Furthermore, by substituting Equation (31) into Equation (32), we obtain Equation (30) and thus Equation (14) for $1 \leq m \leq p-1$. To obtain Equation (13), we substitute $S_{p}=-I$ into Equation (11).

$$
I-\Psi_{0}=-S_{0}-\sum_{m=1}^{p-1} S_{m} \Delta t^{-m}+\Delta t^{-p} I .
$$

By substituting Equation (31) into this equation, we obtain the next formula.

$$
I-\Psi_{0}=(-1)^{p-1} \Delta t^{p} \Psi_{p}\left(\Delta t^{-p} I-\sum_{m=1}^{p-1} S_{m} \Delta t^{-m}-S_{0}\right) .
$$

From Equation (31), $\Psi_{p}$ is regular. Then, we reformulate Equation (37) as follows.

$$
\Delta t^{-p} I-\sum_{m=1}^{p-1} S_{m} \Delta t^{-m}-S_{0}=(-1)^{p-1} \Delta t^{-p} \Psi_{p}^{-1}\left(I-\Psi_{0}\right) .
$$


Thus,

$$
S_{0}=-\sum_{m=1}^{p-1} S_{m} \Delta t^{-m}+\Delta t^{-p} I+(-1)^{p} \Delta t^{-p} \Psi_{p}^{-1}\left(I-\Psi_{0}\right)
$$

Substituting Equation (30) inside the second term of this formula, we obtain Equation (13).

\section{E. THE PROOF OF THEOREM 2}

By substituting Equation (30) into $\sum_{m=1}^{p-1} S_{m} \Delta t^{-m}$, we obtain the following relation.

$$
\sum_{m=1}^{p-1} S_{m} \Delta t^{-m}=\sum_{m=1}^{p-1}(-1)^{m} \sum_{j=m}^{p-1} \frac{j !}{(j-m) ! m !} \Psi_{j}+\Delta t^{-p} \sum_{m=1}^{p-1}(-1)^{p+m-1} \frac{p !}{(p-m) ! m !} I .
$$

To derive $\Psi_{0}$, we rewrite Equation (11) by substituting $S_{p}=-I$ in Assumption 1 as follows:

$$
\Psi_{0}=I-\Delta t^{-p} I+\sum_{m=1}^{p-1} S_{m} \Delta t^{-m}+S_{0}
$$

By substituting Equation (13) and Equation (40) into this equation, we obtain the following.

$$
\begin{aligned}
\Psi_{0}= & I-\Delta t^{-p} I+\sum_{m=1}^{p-1}(-1)^{m} \sum_{j=m}^{p-1} \frac{j !}{(j-m) ! m !} \Psi_{j}+\Delta t^{-p} \sum_{m=1}^{p-1}(-1)^{p+m-1} \frac{p !}{(p-m) ! m !} I+\Delta t^{-p} I \\
& -\sum_{m=1}^{p-1}\left\{(-1)^{m} \sum_{j=m}^{p-1} \frac{j !}{(j-m) ! m !} \Psi_{j}+(-1)^{p+m-1} \Delta t^{-p} \frac{p !}{(p-m) ! m !} I\right\} \\
& +(-1)^{p} \Delta t^{-p} \Psi_{p}^{-1}\left(I-\Psi_{0}\right) \\
= & I+(-1)^{p} \Delta t^{-p} \Psi_{p}^{-1}\left(I-\Psi_{0}\right) .
\end{aligned}
$$

From Equation (4a), we see that $\Phi_{p}=\left(I-\Psi_{0}\right)^{-1} \Psi_{p}$, where $I-\Psi_{0}$ is always regular by Assumption 1 and Equation (11). Since $\Psi_{p}$ is regular by Equation (31), $\Phi_{p}$ is also regular. Thus, we write as $\Phi_{p}^{-1}=\Psi_{p}^{-1}\left(I-\Psi_{0}\right)$. By substituting it into Equation (42), we derive the following expression.

$$
\Psi_{0}=I+(-1)^{p} \Delta t^{-p} \Phi_{p}^{-1},
$$

and thus we obtain Equation (15).

\section{ACKNOWLEDGMENTS}

We would like to express our great appreciation to Prof. Shouhei Shimizu at The Institute of Scientific and Industrial Research, Osaka University for our use of his code to generate the artificial data for the evaluation.

\section{REFERENCES}

P. J. Brockwell and R. A. Davis. 1991. Time Series: Theory and Methods (2nd. ed.). Springer, New York.

M. Demeshko, T. Washio, and Y. Kawahara. 2013. A novel structural AR modeling approach for a continuous time linear Markov system. In Proceedings of the 1st IEEE ICDM Workshop on Causal Discovery 2013 (CD'13).

F. Fisher. 1970. A correspondence principle for simultaneous equation models. Econometrica 38, 1 (1970), 73-92. 
J. Gottschalk. 2001. An Introduction into the SVAR Methodology: Identification, Interpretation and Limitations of SVAR Models. (2001).

D. Hinrichsen and A. J. Pritchard. 2005. Mathematical Systems Theory I, Modelling, State Space Analysis, Stability and Robustness. Vol. 48. Springer.

A. Hyvärinen, S. Shimizu, and P. Hoyer. 2008. Casual modeling combining instantaneous and lagged effects: An identifiable model based on non-Gaussianity. In Proceedings of the 25th International Conference on Machine Learning. 424-431.

Y. Iwasaki and H. A. Simon. 1994. Causality and model abstraction. Artificial Intelligence 67 (1994), $143-194$.

Y. Kawahara, S. Shimizu, and T. Washio. 2011. Analyzing relationships between ARMA processes based on non-Gaussianity of external influences. Neurocomputing 74, 12-13 (2011), 2212-2221.

L. Kilian. 2011. Structural Vector Autoregressions. Edward Elger.

G. Lacerda, P. Spirtes, J. Ramsey, and P. O. Hoyer. 2008. Discovering cyclic causal models by independent components analysis. In Proceedings of the 24th Conference on Uncertainty in Artificial Intelligence.

P. Lax and R. Richtmyer. 1956. Survey of the stability of linear finite difference equations. Communications on Pure and Applied Mathematics 9 (1956), 267-293.

H. Levy and F. Lessman. 1992. Finite Difference Equations. Dover.

A. Moneta, N. Chlaß, D. Entner, and P. O. Hoyer. 2011. Causal search in structural vector autoregressive models. In JMLR Workshop and Conference Proceedings, Causality in Time Series, Vol. 12. 95-118.

A. Moneta, D. Entner, P. Hoyer, and A. Coad. 2010. Causal inference by independent component analysis with applications to micro- and macroeconomic data. Jena Economic Research Papers No. 2010-031 (2010), 3-4.

J. M. Mooij, D. Janzing, and B. Schölkopf. 2013. From ordinary differential equations to structural causal models: The deterministic case. In Proceedings of the 29th Conference on Uncertainty in Artificial Intelligence.

J. Pearl. 2000. Causality: Models, Reasoning, and Inference. Cambridge University Press.

Yu. N. Pepyolyshev. 1988. Spectral characteristics of power noise parameters and fluctuations of neutron reflectors of nuclear reactor IBR-2. (1988). (In Russian).

B. Pfaff and T. Kronberg. 2008. VAR, SVAR and SVEC models: Implementation within R package vars. Journal of Statistical Software 27, 4 (2008), 1-32.

B. L. Shea. 1987. Estimation of multivariate time series. Journal of Time Series Analysis 8 (1987), 95-110.

S. Shimizu, T. Inazumi, Y. Sogawa, A. Hyvärinen, Y. Kawahara, T. Washio, P. O. Hoyer, and K. Bollen. 2011. DirectLiNGAM: A direct method for learning a linear non-Gaussian structural equation model. Journal of Machine Learning Research 12 (2011), 1225-1248.

O. Stamer, R. Tweedie, and P. J. Brockwell. 1996. Existence and stability of continuous time threshold ARMA process. Statistica Sinica 6 (1996), 715-732.

J. Strikwerda. 1989. Finite Difference Schemes and Partial Differential Equations. Chapman and Hall, London.

M. Voortman, D. Dash, and M. J. Druzdzel. 2010. Learning why things change: The difference-based causality learner. In Proceedings of the 26th Conference on Uncertainty in Artificial Intelligence.

Received July 2014; revised October 2014; accepted December 2014 